\title{
THE IMPACT OF RADIODERMATITIS IN QUALITY OF LIFE OF PATIENTS WITH BREAST CANCER DURING TREATMENT: A PROSPECTIVE LONGITUDINAL STUDY
}

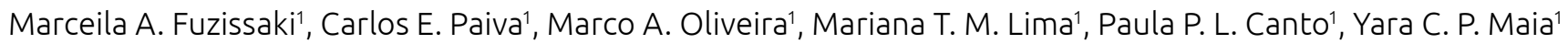
1 Universidade Federal de Uberlândia - Uberlância (MG), Brazil.

Objective: To investigate the influence of radiodermatitis severity on the quality of life (QOL) of women with breast cancer (BC) throughout radiotherapy (RT). Methodology: A prospective longitudinal study conducted with $100 \mathrm{BC}$ patients evaluated weekly during RT and three months after treatment. The questionnaire The Dermatology Life Quality Index (DLQI) and Radiation Therapy Oncology Group (RTOG) Scale were used to evaluate QOL and radiodermatitis, respectively. The Generalized Estimated Equations (GEE) were used to examine the association between the total score of QOL and their domains (symptoms and feelings, daily activities, leisure, personal relationships, work/school and treatment), time of RT and the radiodermatitis score, controlled by confounding factors (age, years of study and economic classification). Estimated marginal means and 95\% confidence intervals were compared pairwise by applying Sequential Sidak for multiple tests. Results: The total QOL score, as well their domains (work/school, leisure, daily activities, symptoms and feelings) were significantly associated with the time of RT ( $\mathrm{p} \leq 0.001)$, with the radiodermatitis score $(\mathrm{p} \leq 0.001)$ and with the interaction time of treatment and radiodermatitis score $(\mathrm{p} \leq 0.001)$. When assessing the post-hoc (sidak sequencial), it was identified that the worst QOL scores were in the presence of grade 3 (mean $=6.00$ ) in T3 and grade $4(6.50 ; 7.00)$ in T5 and T6, respectively, and this difference was statistically significant. Considering the domain signs and symptoms, we recognized a worse score in the presence of grade 4 (3.00) in T6. Regarding to the work and school domains and daily activities, the worst scores were identified in T5, in patients with grade 0 (4.00) and grade 3 (2.50), respectively. The leisure domain had a worse score in T3 in grade 3 (3.00). Conclusion: Women with BC had a negative impact on QOL during RT, with the greatest impact related to severe radiodermatitis. Actions aimed at minimizing the impairment in QOL need to be adopted to transform this exhausted process, less traumatic and easier to finalize. 